\title{
Transzonular vitreous injection vs a single drop compounded topical pharmaceutical regimen after cataract surgery
}

This article was published in the following Dove Press journal:

Clinical Ophthalmology

18 July 2016

Number of times this article has been viewed

\author{
Bret L Fisher ${ }^{\prime}$ \\ Rick Potvin ${ }^{2}$ \\ 'Eye Center of North Florida, Panama \\ City, FL, USA; ${ }^{2}$ Science in Vision, \\ Akron, NY, USA
}

Video abstract

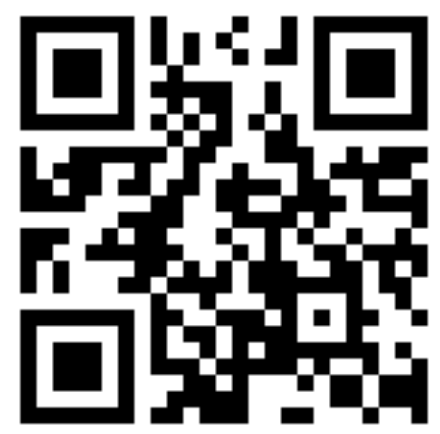

Point your SmartPhone at the code above. If you have a QR code reader the video abstract will appear. Or use

http://youtu.be/SLOIYpHvyFo
Correspondence: Bret L Fisher Eye Center of North Florida, 2500 Martin Luther King Blvd,

Panama City, FL 32405, USA

$\mathrm{Tel}+\mathrm{I} 8507843937$

$\mathrm{Fax}+\mathrm{I} 8505229829$

Email bfisher@eyecarenow.com
Purpose: To compare the relative effectiveness of a Tri-Moxi-Vanc intraocular solution injected transzonularly into the vitreous with the topical formulation of Pred-Moxi-Ketor (given for the first week postoperatively) followed by Pred-Ketor (given for weeks 2-4 after surgery).

Patients and methods: This was a single-site, single-surgeon, prospective, randomized, subjectmasked contralateral eye study with an active comparator and was approved by an appropriate ethics committee. Twenty-five subjects with uncomplicated cataract who were scheduled for cataract surgery were enrolled. If surgery was uneventful, subjects received either an injection in that eye or followed a minimum drop postoperative pharmaceutical regimen. The second eye surgery was performed with the opposite treatment. Subjects were followed for 1 month. Measures of interest were the changes in intraocular pressure (IOP) from baseline and the changes in corneal and macular thickness. Subjects were also asked to evaluate pain perception, visual quality, and overall satisfaction with surgery. They were also asked which regimen they preferred.

Results: IOP was not statistically significantly different between the groups $(P=0.81)$; there was also no statistically significant difference in IOP over time $(P=0.74)$. There was no statistically significant difference in central macular thickness at 1 week and 1 month between the groups $(P=0.18)$. The central corneal thickness was significantly greater 1 day postoperatively relative to baseline, but there was no statistically significant difference between the groups at any time point $(P=0.92)$. The difference in reported pain was also not statistically significantly different between the groups $(P=0.67)$. Satisfaction with surgery was similar for both groups, but significantly more subjects preferred the injection for overall experience $(P<0.01)$.

Conclusion: Cataract surgery completed with the two pharmaceutical regimens was similar in outcome. Significantly more subjects preferred the injection, presumably as a function of the greater convenience with no apparent difference in the therapeutic effect.

Keywords: prophylaxis, phacoemulsification, intracameral, antibiotics, steroids, cataract surgery

\section{Introduction}

Appropriate prophylaxis at the time of cataract surgery can contribute to a safe and quick visual recovery. Prophylactic pharmaceutical regimens are designed to decrease the likelihood and severity of infection, inflammation, and postoperative pain. They may begin before surgery and continue in the postoperative period. Prophylactic medications are typically administered topically or using intraocular injection at the time of surgery, though postoperative prophylaxis is generally restricted to topical application. Noncompliance is a significant issue; noncompliance with ophthalmic topical drops has been reported to be as high as $40 \% .^{1}$ Noncompliance is likely to increase the risk of infection, and may pose a health safety concern; for instance, not 
adhering to the prescribing schedule for antibiotic eyedrops could increase the risk of bacterial resistance.

After cataract surgery, a patient may be instructed to instill one or more antibiotic drops, additional drops for inflammation, and possibly other drops for pain management; these drops are often instilled on different schedules. It can be confusing for patients, which can contribute to noncompliance. A partial solution to the issue is to combine multiple therapies into a single drop. In addition to improving compliance, the one-drop combination may lower ocular toxicity by decreasing preservative concentration and reduce drug dilution by eliminating consecutive drop instillations. ${ }^{2}$ Studies have shown that a combination of antibiotic/steroid eyedrops provided similar results to the noncombined drops after cataract surgery. ${ }^{2,3}$ One available multiple drop formulation is a topical formulation (Less Drops ${ }^{\mathrm{TM}}$; Imprimis Pharmaceuticals Inc., San Diego, CA, USA) consisting of Pred-Moxi-Ketor and Pred-Ketor. As the names suggest, the Pred-Moxi-Ketor contains prednisolone, moxifloxacin, and ketorolac, whereas the Pred-Ketor contains prednisolone and ketorolac. Moxifloxacin is an effective topical antibacterial agent that has been demonstrated to lower the endophthalmitis rate after cataract surgery. ${ }^{4}$ Prednisolone is commonly used for managing postoperative inflammation. ${ }^{5}$ Ketorolac is a nonsteroidal anti-inflammatory drug (NSAID) shown to effectively decrease macular edema, ${ }^{6}$ inflammation, and pain postoperatively. ${ }^{7}$ The effectiveness of the NSAID in providing significant improvement in macular edema or visual outcomes is less apparent in patients at low risk for complications when compared to placebo. ${ }^{8}$

The use of single therapy drop does simplify things for the patient, but they are still required to administer the drop several times a day. In addition, it is possible that the penetration of these topical drops through the cornea may be insufficient to provide the required benefit. ${ }^{9}$

An alternative to topical medication after cataract surgery is an intraocular injection. The medication is delivered directly into the eye, so penetration is not an issue. The rate of endophthalmitis was reported to be $0 \%$ in 25,920 subjects given intracameral antibiotics, whereas the rate was higher with other delivery methods, suggesting the former was the best delivery method. ${ }^{10}$ An additional study reported fewer anterior chamber cells 1 month postoperatively in a group receiving an injection of triamcinolone and gentamicin with topical tobramycin drops when compared with a group receiving topical dexamethasone and tobramycin eyedrops. ${ }^{11}$ Some have argued that intraocular injection after uneventful cataract surgery carries its own risks, including the potential for toxicity and for higher antibacterial resistance, which may offset the benefits of reducing the already low rate of cataract surgery complications. ${ }^{12,13}$ However, a meta-analysis evaluating 18 large studies concluded that intracameral antibacterial injections are more effective at reducing the likelihood of endophthalmitis when compared with topical eyedrops. ${ }^{14}$

An injection currently available for use after cataract surgery is the Tri-Moxi-Vanc intraocular solution (Dropless ${ }^{\mathrm{TM}}$; Imprimis Pharmaceuticals Inc.). This drug formulation includes triamcinolone, moxifloxacin, and vancomycin. It is designed to be injected transzonularly into the vitreous. Triamcinolone has been shown to produce similar results when compared with topical steroid administration in terms of postoperative cells and flare, intraocular pressure (IOP), best-corrected visual acuity (BCVA), conjunctival injection, symptoms, and complications. ${ }^{15,16}$ Intracameral moxifloxacin injection reduced endophthalmitis without reports of toxic anterior segment syndrome or increased corneal endothelial cell loss. ${ }^{17,18}$ The introduction of intracameral vancomycin has been demonstrated to reduce the risk of endophthalmitis by a factor of 37.5, ${ }^{19}$ without a significant negative impact on the average central retinal thickness or contrast sensitivity. ${ }^{20}$

The purpose of this study was to compare the relative effectiveness of the injection with the topical formulation of PredMoxi-Ketor (given for 1 week postoperatively) followed by Pred-Ketor (given for weeks 2-4 after surgery). The primary interest was to determine if there were differences in IOP changes postoperatively between the two treatments. Other differences investigated were related to postoperative healing, visual quality, patient satisfaction, and regimen preference.

\section{Patients and methods}

This was a single-site, single-surgeon, prospective randomized, subject-masked contralateral eye study with an active comparator (NCT02819908). The study was reviewed and approved by an appropriate ethics review board (Salus IRB, Austin, TX, USA). The study involved 25 subjects implanted binocularly (one eye at a time) at one site. Subjects served as their own controls, with randomization of the two procedures by eye (left/ right) and by surgery order (first eye/second eye). Half of the subjects received the injection in their first eye. When the second eye surgery was performed, typically within 2 weeks, they followed the pharmaceutical regimen described in the following paragraphs. The remaining patients followed the pharmaceutical regimen after first eye surgery and had the injection in their second eye. The study was conducted in a manner consistent with the Declaration of Helsinki and Good Clinical Practice.

Eligible subjects were patients of either sex, over the age of 21, presenting for uncomplicated bilateral cataract surgery or refractive lens exchange surgery with intraocular lens 
implantation where surgery in each eye was scheduled between 1 and 2 weeks apart. Potential subjects were provided information on the study and asked to sign a patient information and consent form to participate. Subjects had to demonstrate a potential postoperative acuity of $20 / 30$ or better $(0.2 \log$ MAR $)$ in each eye. Preoperative exclusion criteria included ocular pathology such as amblyopia, proliferative diabetic retinopathy, macular edema, uveitis or chronic ocular inflammation, and other conditions likely to affect postoperative visual acuity and/or the normal course of postoperative healing. Each subject had a standard preoperative workup that included measurement of IOP and central macular thickness.

The use of epinephrine or antibiotics in the bottle was not permitted. Complications during surgery, such as vitreous loss, anterior chamber hyphema, or capsular rupture were exclusion criteria, as was the inability to deliver the full intracameral dose into the eye for the injection treatment. These operative events were recorded, and the subject was removed from the effectiveness part of the study, though followed for safety purposes.

The surgeon's standard technique, which included the use of povidone-iodine for antisepsis, was used for all surgeries, with the same ophthalmic viscosurgical device used for all cases. Subjects were randomized such that the first eye received either the injection or the postoperative pharmaceutical regimen described in the following paragraphs. There was no postoperative pharmaceutical regimen for eyes randomized to the injection at the time of surgery.

In the subjects receiving the "Dropless ${ }^{\text {TM" }}$ regimen, $0.2 \mathrm{cc}$ of the Tri-Moxi-Vanc formulation was instilled into the anterior vitreous space using a 27-gauge blunt curved cannula. With the eye filled with viscoelastic, this cannula is introduced through the temporal clear corneal cataract surgery incision, and then directed by the operating surgeon beneath the iris nasally and over the peripheral edge of the lens capsule. The tip of the cannula then passes between the zonular fibers, where the medication is then slowly injected. The medication in most cases can be visualized as it enters the anterior vitreous space posterior to the lens implant. Once injected, the medication remains sequestered in the anterior vitreous space, even as viscoelastic is subsequently removed from the eye.

For eyes randomized to the topical drops regimen, the formulation of Pred-Moxi-Ketor was prescribed three times a day for 1 week postoperatively, followed by Pred-Ketor prescribed two times a day for weeks 2-4 after surgery. Subjects were asked to record their drop usage in a diary provided for that purpose.

Subjects were seen for evaluation 1 day, 1 week, and 1 month after surgery for the given eye. Each postoperative exam included a standard slit-lamp examination with evaluation of cells and flare. Other tests included IOP measurement, refraction (except day 1) and visual acuity, anterior chamber and retinal tomography, as well as evaluations of patient satisfaction, patient preference, pain, and visual symptoms. Specific attention was paid to rebound inflammation, diagnosed based on symptoms of ocular pain, blurry vision, light sensitivity and redness, and slit-lamp examination showing anterior chamber cells and flare. Rebound inflammation was managed with appropriate additional medications. All adverse events were recorded and monitored.

Clinical data were tabulated and deidentified on appropriate case report forms. The data were imported into an MS Access database for data checking, collation, and preliminary analysis (Microsoft Corp., Redmond, WA, USA). Statistical analyses were performed using the STATISTICA data analysis software system, version 12 (StatSoft, Inc., Tulsa, OK, USA). Statistical testing was performed using analysis of variance (ANOVA) on continuous variables and appropriate nonparametric tests on categorical data. Statistical significance was set at $P=0.05$.

\section{Results}

A total of 25 subjects were successfully recruited, 16 females and nine males; all subjects were Caucasian. One subject was unable to attend their 1 month visit for their second eye surgery, but all other visits were completed. The average age

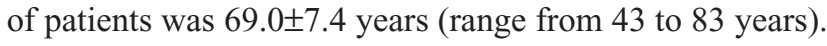
The majority of cataracts were Grade 2 or 3 nuclear sclerosis, with no difference between the two groups. Surgery was uneventful in all cases, with no difference in the cumulative distributed energy (ANOVA, $P=0.67$ ) or fluid used (ANOVA, $P=0.36$ ) between groups. Transzonular injection was completed successfully in all eyes in the injection group.

IOP was compared to baseline at the 1 day, 1 week, and 1 month postoperative visits. Figure 1 shows the average change from baseline in the two groups. A repeated measures ANOVA showed no statistically significant difference between the groups $(P=0.81)$, no statistically significant difference in IOP over time $(P=0.74)$, and no group/time interaction.

Figure 2 shows the average change in central corneal thickness by group over time. The central corneal thickness was significantly higher at day 1 postoperatively for both groups $(P<0.01)$, but there was no statistically significant difference between the groups $(P=0.92)$ at any time point. In both groups, the central corneal thickness had returned to preoperative levels by the 1-month visit.

The central macular thickness was also measured at 1 week and 1 month. There was no statistically significant 


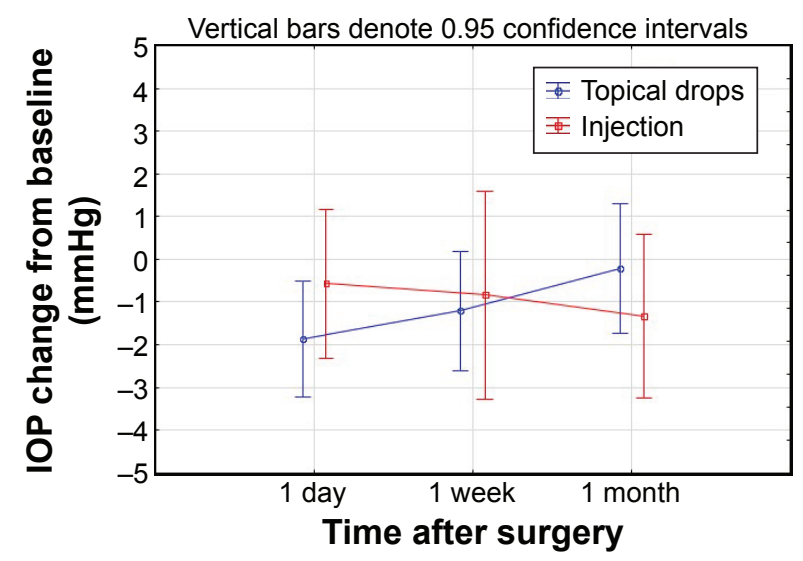

Figure I Change in IOP over time by group. Abbreviation: IOP, intraocular pressure.

difference between the groups at either time point $(P=0.18)$, though the thickness was statistically significantly higher in both groups at 1 month $(265 \pm 21 \mu \mathrm{m}$ preoperative vs $273 \pm 25 \mu \mathrm{m}$ postoperative, $P<0.01$ ).

Figure 3 shows the BCVA of subjects preoperatively and at 1 week and 1 month postoperative. There was no statistically significant difference between the groups at any time point, though BCVA was statistically significantly better after surgery $(P<0.01)$, improving about 2 lines on average in both groups.

Pain was reported by only six patients after surgery, associated with four eyes in the topical drops group and two eyes in the injection group; the difference was not statistically significantly different between the groups $(P=0.67)$. No subject in the injection group reported pain the day after surgery, whereas three subjects reported pain on day 1 in the topical drops group. In all but one topical drops case, the pain was reported as transient and mild. The one case of severe pain at 1 week was reported as mild at 1 month.

Floaters were observed by only one subject, in the injection group, the day after surgery and not on any subsequent visits; the floaters were reported as mild and infrequent.

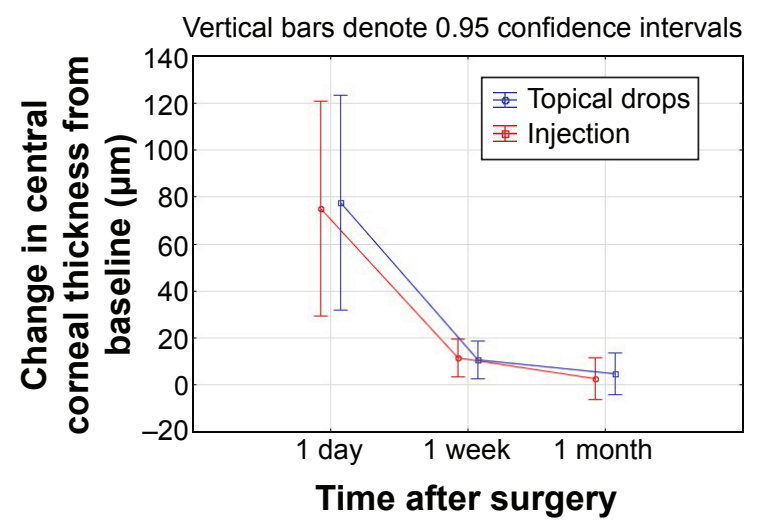

Figure 2 Central corneal thickness change over time by group.

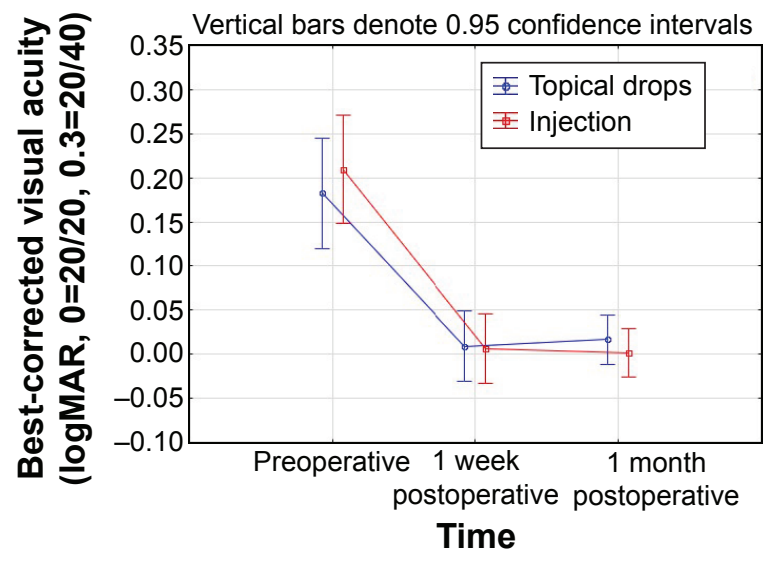

Figure 3 Best-corrected visual acuity over time by group.

Although cells and flare were evident in all patients on the day after surgery, there was no difference in the severity of either by group $(P>0.50)$. The cells and flare resolved over time, again with no significant difference between the groups.

Subject satisfaction was measured at the 1 week and 1 month visits after each surgery. The results of the 1-month follow-up visit for the second eye are of most interest because both eyes had been treated at that point. Randomization of eyes ensured that a near-equal number of eyes receiving an injection (13) and topical drops (12) were the second eye treated. Of the 24 subjects completing all visits, 20 (83\%) reported being "Very Satisfied", with two more "Satisfied", and two "Neither Satisfied nor Dissatisfied". No subject was dissatisfied. Evaluating the overall surgical experience, 22 of $24(92 \%)$ indicated they preferred the injection, with the other two preferring the topical drops regimen. In terms of evaluating the postoperative visual outcome, 21 of 24 (88\%) preferred the injection, two preferred the topical drops regimen, and one felt the two were equal.

There were five study-related minor adverse events reported, all of which were rebound inflammation. All cases were treated with additional medication and resolved without sequelae. Two cases were in the topical drops group and three in the injection group, with one subject affected bilaterally. There was no statistically significant difference in the incidence of rebound inflammation by treatment group $(P=0.68)$.

\section{Discussion}

To the best of our knowledge, this is the first study to compare the combination of both anti-infective and antiinflammatory drugs using transzonular injection and topical delivery methods for the purpose of cataract surgery prophylaxis. The results in the current study suggest that patients receiving the combination drops in one eye and a one-time intraocular injection in the other eye had similar satisfaction 
and outcomes, though more patients preferred the injection to the drops. One other study examined similar groups but included topical antibiotic application in the injection group, and the formulation was slightly different than the one used in the current study. In that study, the authors ${ }^{11}$ found that postsurgical inflammation (based on anterior chamber cell counts) was similar to or lower in the injection group when compared to the drops group. The addition of topical antibiotics to the injection group is likely unnecessary, although it has been a topic of debate. ${ }^{21,22}$

It should be noted that transzonular injection into the anterior vitreous is very different from intracameral injection, where medications are simply injected through a side port incision into the anterior chamber of the eye. In the latter case, the drugs remain in the eye for only a short period before being washed out by the natural ongoing production and filtration of aqueous solution. In the case of tranzonular medication delivery into the vitreous space, the medication, much like with intravitreal injections given through the pars plana, remains within the matrix of the vitreous body itself for a prolonged period, releasing over a longer time. With this approach, most patients require no further medication, either antibiotic or anti-inflammatory, to heal successfully and completely from their cataract surgery.

Steroid and NSAID prophylaxis after cataract surgery are intended to decrease inflammation and provide comfort without significantly increasing IOP. The relevant components here were the triamcinolone injection in one group and the topical prednisolone and ketorolac drops in the other group. In this study, both treatments were similar in effectively reducing inflammation without significantly increasing IOP. Patient symptoms, particularly related to pain, did not significantly differ between the two groups. These results are consistent with findings in previous studies. ${ }^{15,16}$ For instance, a group injected with triamcinolone acetonide had similar visual acuity, inflammation, mean IOP, and symptoms when compared with a group using topical prednisolone acetate, with no change in the risk for endophthalmitis. ${ }^{15}$ In a second study, injecting an effective dose of triamcinolone acetonide followed by $250 \mathrm{mg}$ acetazolamide provided statistically similar BCVA, flare, and IOP without significantly increasing complication risks when compared with the group receiving Betnesol-N eyedrops (contain the steroid betamethasone and the antibiotic neomycin); all patients in this study were also injected with cefuroxime and betamethasone subconjunctivally. ${ }^{16}$

The antibiotics used in the current injection group (moxifloxacin and vancomycin) have been reported to be both safe and effective in previous studies. ${ }^{17-20}$ Although many previous studies have included intracameral cefuroxime injection instead of moxifloxacin, the latter has been shown to be equally effective. ${ }^{21}$ Vancomycin was associated with a significant reduction in endophthalmitis rates in a large multiyear study. ${ }^{19}$ A number of additional studies suggest that antibiotic injections may be more effective than topical antibiotics for the prevention of endophthalmitis after cataract surgery, though endophthalmitis rates in both scenarios are low. ${ }^{10,14,21}$ While the combination of moxifloxacin and vancomycin used in the current study is likely to be very effective against almost all endophthalmitis-causing bacteria, some have raised concerns regarding possible bacterial drug resistance. However, Braga-Mele et $\mathrm{al}^{22}$ point out in a recent review that the anterior chamber is a relatively closed system, hence it is "unlikely that routine intracameral antibiotic prophylaxis will promote drug resistance".

Damage to the corneal endothelium and subsequent increase in corneal thickness are often associated with trauma during cataract surgery; toxicity from intracameral drugs is another possible cause. Intracameral moxifloxacin has not been noted to change endothelial cell count when compared with a group that did not receive intracameral moxifloxacin. ${ }^{17}$ In the current study, the lack of any significant difference between the two study groups in terms of central corneal thickness and BCVA suggests the injected drug formulation does not present a toxicity risk to the corneal endothelium. Concerns regarding toxicity or adverse events with injectable drugs are often related to dosage or preparation errors. ${ }^{16,22}$ The controlled concentration and the pre-prepared formulations used here reduce that risk.

Postoperative complications after vancomycin injection, specifically including macular edema, have been reported, ${ }^{12,19}$ although another study suggests that vancomycin is unlikely to significantly increase macular edema or decrease visual function..$^{20}$ Our results are consistent with the latter finding, as macular edema was not significantly different in the two groups of eyes. The increase in macular thickness 1 month after surgery, observed in the current study, is consistent with values reported previously. ${ }^{8}$

Patient satisfaction with surgery in both groups was high, with 22 of 24 subjects being satisfied or very satisfied with their outcome. Despite the similarity in satisfaction between the two groups, the majority of patients $(-90 \%)$, when asked about their overall postoperative experience and vision/ outcomes, preferred the injection to the self-administration of drops. This is presumably a function of the fact that there was no perceived difference in the results for both eyes, but the injection eliminated the need for any postoperative drop regimen. While not stated by study subjects, the injection 
also removes any risk related to noncompliance with the prescribed pharmaceutical regimen.

A limitation to the study was the sample size. The study was powered to allow for detection of a mean difference in IOP of $3 \mathrm{mmHg}$ with an $\alpha$ of 0.05 and a power of 0.9 , presuming that the standard deviation of Goldmann tonometry was $3 \mathrm{mmHg}$. For other measures, such as signs of inflammation, a larger sample size would have been helpful.

\section{Conclusion}

Cataract surgery completed with the use of prophylactic anti-infective and anti-inflammatory drugs delivered transzonularly into the vitreous or topically provided similar safety in terms of IOP and corneal and macular edema, as well as similar effectiveness in terms of inflammation control, visual acuity, and patient comfort. When subjects were asked about their preference, the majority chose the drug injection over the drop administration, despite being similarly satisfied with the results in each eye. This is presumably a function of increased convenience with no apparent compromise in therapeutic effect.

\section{Acknowledgments}

The Eye Center of North Florida received an investigatorinitiated study grant from Imprimis Pharmaceuticals to conduct this research. The Eye Center of North Florida provided funding to Science in Vision to assist with data analysis and preparation of this manuscript. Sarah Y Makari, OD, a consultant to Science in Vision, received compensation for providing writing assistance to the authors in preparation of the manuscript.

\section{Disclosure}

The authors report no conflicts of interest in this work.

\section{References}

1. Vandenbroeck S, De Geest S, Dobbels F, Fieuws S, Stalmans I, Zeyen T. Prevalence and correlates of self-reported nonadherence with eye drop treatment: the Belgian Compliance Study in Ophthalmology (BCSO). J Glaucoma. 2011;20(7):414-421.

2. Cunha PA, Shinzato FA, Tecchio GT, Weber SL, Brasil A, Avakian A. Efficacy and tolerability of a gatifloxacin/prednisolone acetate fixed combination for topical prophylaxis and control of inflammation in phacoemulsification: a 20-day-double-blind comparison to its individual components. Clinics (Sao Paulo). 2013;68(6):834-839.

3. Freitas LL, Soriano E, Muccioli C, Höfling-Lima AL, Belfort R Jr. Efficacy and tolerability of a combined moxifloxacin/dexamethasone formulation for topical prophylaxis and reduction of inflammation in phacoemulsification: a comparative, double masked clinical trial. Curr Med Res Opin. 2007;23(12):3123-3130.
4. Rudnisky CJ, Wan D, Weis E. Antibiotic choice for the prophylaxis of post-cataract extraction endophthalmitis. Ophthalmology. 2014;121(4): 835-841.

5. Lorenz K, Dick B, Jehkul A, Auffahrt GU. Inflammatory response after phacoemulsification treated with $0.5 \%$ prednisolone acetate or vehicle. Graefes Arch Clin Exp Ophthalmol. 2008;246(11):1617-1622.

6. Wittpenn JR, Silverstein S, Heier J, Kenyon KR, Hunkeler JD, Earl M; Acular LS for Cystoid Macular Edema (ACME) Study Group. A randomized, masked comparison of topical ketorolac $0.4 \%$ plus steroid vs steroid alone in low-risk cataract surgery patients. Am J Ophthalmol. 2008;146(4):554-560.

7. Donnenfeld ED, Nichamin LD, Hardten DR, et al. Twice-daily, preservative-free ketorolac $0.45 \%$ for treatment of inflammation and pain after cataract surgery. Am J Ophthalmol. 2011;151(3):420-426.

8. Tzelikis PF, Vieira M, Hida WT, et al. Comparison of ketorolac $0.4 \%$ and nepafenac $0.1 \%$ for the prevention of cystoid macular oedema after phacoemulsification: prospective placebo-controlled randomised study. Br J Ophthalmol. 2015;99(5):654-658.

9. Donnenfeld ED, Comstock TL, Proksch JW. Human aqueous humor concentrations of besifloxacin, moxifloxacin, and gatifloxacin after topical ocular application. J Cataract Refract Surg. 2011;37(6):1082-1089.

10. Jabbarvand M, Hashemian H, Khodaparast M, Jouhari M, Tabatabaei A, Rezaei S. Endophthalmitis occurring after cataract surgery: outcomes of more than 480000 cataract surgeries, epidemiologic features, and risk factors. Ophthalmology. 2016;123(2):295-301.

11. Simaroj P, Sinsawad P, Lekhanont K. Effects of intracameral triamcinolone and gentamicin injections following cataract surgery. $J$ Med Assoc Thai. 2011;94(7):819-825.

12. Witkin AJ, Shah AR, Engstrom RE, et al. Postoperative hemorrhagic occlusive retinal vasculitis: expanding the clinical spectrum and possible association with vancomycin. Ophthalmology. 2015;122(7):1438-1451.

13. Schimel AM, Alfonso EC, Flynn HW Jr. Endophthalmitis prophylaxis for cataract surgery: are intracameral antibiotics necessary? JAMA Ophthalmol. 2014;132(11):1269-1270.

14. Kessel L, Flesner P, Andresen J, Erngaard D, Tendal B, Hjortdal J. Antibiotic prevention of postcataract endophthalmitis: a systematic review and meta-analysis. Acta Ophthalmol. 2015;93(4):303-317.

15. Karalezli A, Borazan M, Akova YA. Intracameral triamcinolone acetonide to control postoperative inflammation following cataract surgery with phacoemulsification. Acta Ophthalmol. 2008;86(2):183-187.

16. Negi AK, Browning AC, Vernon SA. Single perioperative triamcinolone injection versus standard postoperative steroid drops after uneventful phacoemulsification surgery: randomized controlled trial. $J$ Cataract Refract Surg. 2006;32(3):468-474.

17. Matsuura K, Miyoshi T, Suto C, Akura J, Inoue Y. Efficacy and safety of prophylactic intracameral moxifloxacin injection in Japan. J Cataract Refract Surg. 2013;39(11):1702-1706.

18. Haripriya A, Chang DF, Namburar S, Smita A, Ravindran RD. Efficacy of intracameral moxifloxacin endophthalmitis prophylaxis at Aravind Eye Hospital. Ophthalmology. 2016;123(2):302-308.

19. Anijeet DR, Palimar P, Peckar CO. Intracameral vancomycin following cataract surgery: an eleven-year study. Clin Ophthalmol. 2010;4: 321-326.

20. Ball JL, Barrett GD. Prospective randomized controlled trial of the effect of intracameral vancomycin and gentamicin on macular retinal thickness and visual function following cataract surgery. $J$ Cataract Refract Surg. 2006;32(5):789-794.

21. Herrinton LJ, Shorstein NH, Paschal JF, et al. Comparative effectiveness of antibiotic prophylaxis in cataract surgery. Ophthalmology. 2016;123(2):287-294.

22. Braga-Mele R, Chang DF, Henderson BA, Mamalis N, Talley-Rostov A, Vasavada A; ASCRS Clinical Cataract Committee. Intracameral antibiotics: safety, efficacy, and preparation. J Cataract Refract Surg. 2014; 40(12):2134-2142. 
Clinical Ophthalmology

\section{Publish your work in this journal}

Clinical Ophthalmology is an international, peer-reviewed journal covering all subspecialties within ophthalmology. Key topics include: Optometry; Visual science; Pharmacology and drug therapy in eye diseases; Basic Sciences; Primary and Secondary eye care; Patien Safety and Quality of Care Improvements. This journal is indexed on

Submit your manuscript here: http://www.dovepress.com/clinical-ophthalmology-journal

PubMed Central and CAS, and is the official journal of The Society of Clinical Ophthalmology (SCO). The manuscript management system is completely online and includes a very quick and fair peer-review system, which is all easy to use. Visit http://www.dovepress.com/ testimonials.php to read real quotes from published authors. 